\title{
Maxillofacial Radiology 184
}

SADJ September 2020, Vol. 75 No. 8 p459

\section{CJ Nortjé}

Below are clinical pictures and images of a very rare lesion presenting in the jaws at birth of a female. The patient spend some time in an incubator (Fig.1). Tracheostomy was a lifesaving procedure in this patient. Repeated operations have removed the gross mass hamartomatous tissue but Figure 2 still shows noticeable recurrence of the lesion. Figure $3 \mathrm{~A}$ and $\mathrm{B}$ are study models at two and a half years of age. The jawbones have grown in size. There is a pronounced shift in the midline of both jaws and their dentitions towards the right and away from the lesion. What are the important radiological findings?
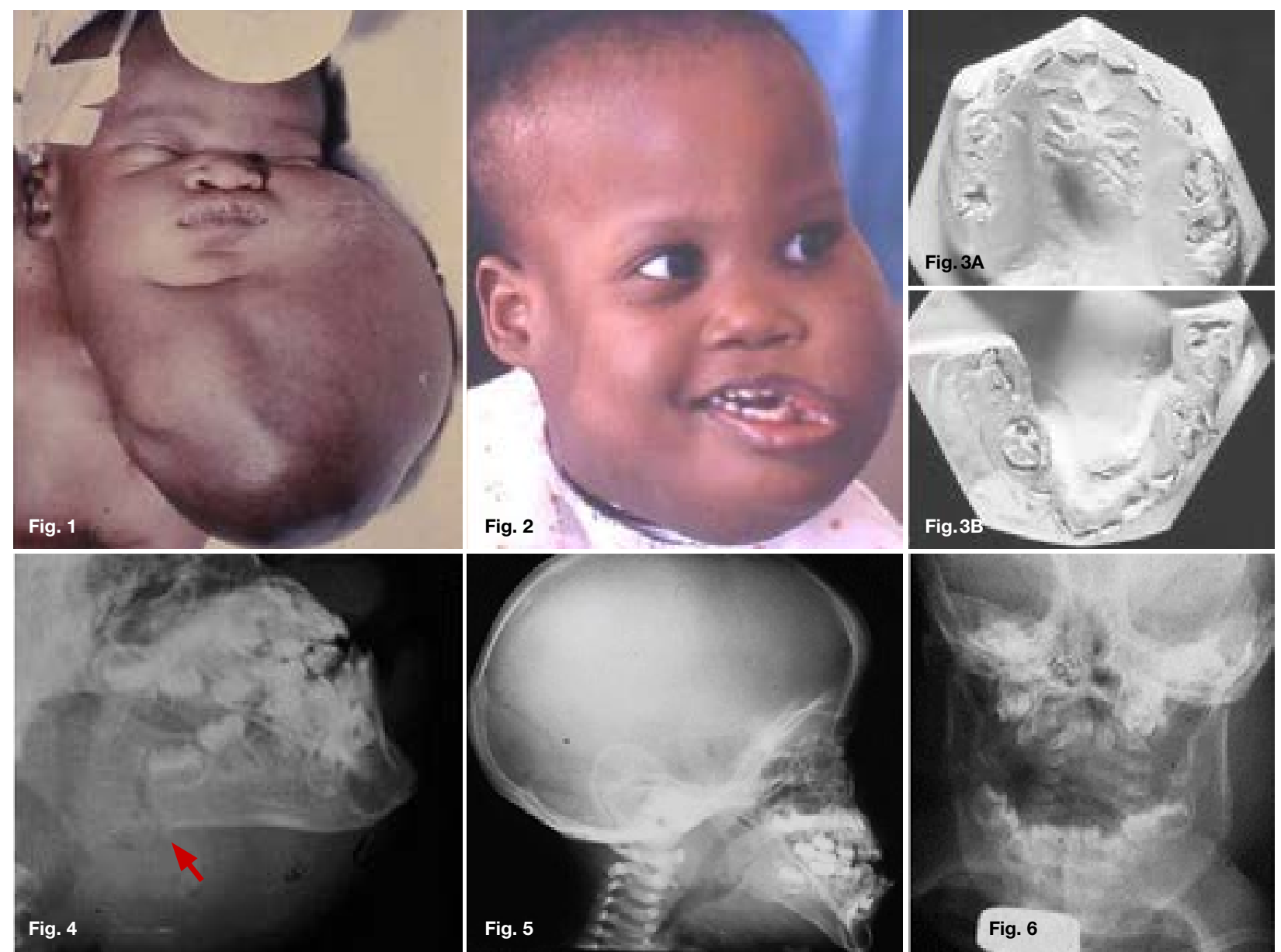

\section{INTERPRETATION}

The lateral oblique radiograph (Fig. 4) of the patient shows an irregularity (red arrow) in the outline of the lower border of the mandible just anterior to the angle. The lateral cephalograph (Fig. 5) show that the deciduous molars are in a Class III occlusal relationship, but the patient has a Class II skeletal pattern. The postero-anterior scull radiograph (Fig. 6) of the patient shows that the lesion is much smaller following repeated surgery but the mandibular asymmetry remains without any reduction in intensity or strength. A histological diagnosis of cervical cystic lymphangioma (Cystic Hygroma) was made. The cystic hygroma are one of the most commonly presenting lymphangiomas.

Christoffel J Nortjé: $B C h D, P h D, A B O M R, D S c$. Faculty of Dentistry, University of the Western Cape.

ORCID Number: 0000-0002-9717-5514

Email: cnortje@uwc.ac.za
They are benign congenital malformations of the lymphatic drainage system that typically form in the neck, clavicle, and axillary regions. Although several series of cystic hygromas have been reported little is known little about the effects this condition has on the mandibulo-facial region. Mal-development of the jaws and consequent dental malocclusion is a possible complication of the condition. In planning the treatment of such patients, maxillofacial surgeons and orthodontists should be consulted at an early stage. Treatment of choice is complete excision, but where vital structures are involved repeated sub-total excisions are advocated.

\section{Reference}

1. Farman AG, Katz, Eloff J, Cywes S. Mandibulo-Facial aspects of the Cervical Cystic Lymphangioma (Cystic Hygroma). British Journal of Oral Surgery. 1978-79; 16: 125-34. 